\title{
Buckling of simply supported GLARE cylindrical panels subjected to uniform compression
}

\author{
Costas Kalfountzos ${ }^{1 *}$, George Bikakis ${ }^{1}$, and Efstathios Theotokoglou ${ }^{1}$ \\ ${ }^{1}$ National Technical University of Athens, Department of Mechanics, GR 15773 Zografou, Greece
}

\begin{abstract}
In this article, the elastic buckling behaviour of cylindrical GLARE (GLAss REinforced) panels with classically simply supported boundary conditions under uniaxial compression is investigated using the finite element method (FEM) and eigenvalue buckling analysis. The buckling coefficient-curvature parameter diagrams of five GLARE grades are obtained and studied along with the diagrams of two glass-fiber composites and monolithic 2024-T3 aluminum, using validated FEM models. It is found that aluminium has a stronger impact on the buckling behaviour of the GLARE panels than the composite layers. From the constructed buckling coefficient - curvature parameter diagrams in double logarithmic scale it is found that there is an approximately linear relation between the buckling coefficient and the curvature parameter of the panels. Based on this finding, appropriate regressions are implemented in order to derive approximate analytical formulas of the buckling coefficient as a function of the curvature parameter for the considered materials.
\end{abstract}

\section{Introduction}

Fiber Metal Laminates (FMLs) are hybrid composite materials built up from thin metal alloy sheets bonded into one laminate with intermediate fibre/epoxy layers. The manufacturing of FMLs has been invented and developed at Delft University of Technology [1]. ARALL (Aramid Reinforced ALuminum Laminate) was the first candidate for aerospace applications, in the late seventies. Later, CARALL (CArbon Reinforced ALuminum Laminate) was investigated, which although it was much stiffer, flight simulation fatigue tests at elevated stress levels resulted in poor fatigue performance due to fiber failure. In 1990, high strength glass fibers were used creating GLARE (GLAss REinforced), which is the most successful FML up to now. GLARE laminates have been selected for many aerospace applications such as the upper fuselage skin material of the Airbus A380 and the cargo floor of Boeing 777 [2].

The elastic buckling of thin panels is a classical problem of the strength of materials with great practical importance and it must always be considered during the design of many engineering applications where stiffened thin-walled structures are employed. For example, the fuselage and wing skin of aerospace structures consist of stringer stiffened curved plates

* Corresponding author: koskalf@yahoo.gr 
which have the shape of cylindrical panels between stringers. FMLs are mainly used for the construction of stiffened thin-walled fuselage structures and, as a result, the investigation of the buckling strength of FML panels is very important. The present study deals with the elastic buckling of thin cylindrical GLARE panels subjected to uniaxial compression on their curved edges. The main objective is the calculation of elastic buckling coefficients of GLARE panels and the construction of pertinent diagrams of the buckling coefficient versus the curvature parameter of the panels. The classical simply supported boundary conditions [3] are considered for the analyzed FMLs.

\section{Problem Definition}

A thin circular cylindrical panel is considered under uniform axial compression consisting of alternating layers of 2024-T3 aluminum and unidirectional (UD) glass-epoxy composite bonded to form GLARE. Fig.1 illustrates the symmetric stacking sequence of a GLARE panel consisting of three aluminum layers (central and external layers) and four cross-ply glass-epoxy layers (among the aluminum layers) along with the applied uniaxial compressive loading. The geometry of the panels is determined as follows: length $L$, radius of curvature $R$, width $b<L$ and panel angle $\varphi$. The thickness $t$ of the panel is very small in comparison with its length and width, and the $R / t$ ratio is always greater than 165 yielding a shallow panel since it is well above the pertinent limit of $R / t=50$ [4]. As shown in Fig.1, for the FEM simulation the plate is loaded statically with a uniaxial constant compressive load $N_{z}$ along the curved edges. Since the axial loads are equal and opposite and the panel is allowed to deform axially because of the applied boundary conditions (bc), it will buckle for a specific value of the applied load $N_{z}$.

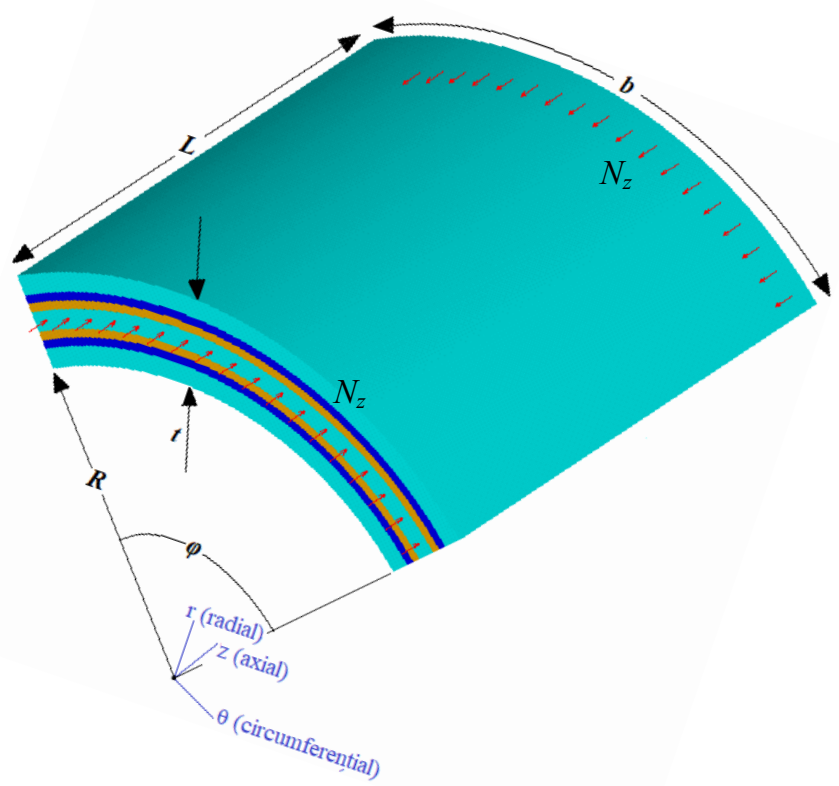

Fig. 1. Geometry of cylindrical GLARE panel under uniform axial compression. 
The classical eigenvalue buckling analysis of ANSYS FEM software [5] is employed in order to calculate the critical buckling load $N_{z c}$. The buckling coefficient $k$ will be determined from the FEM results for variable values of the panel's curvature parameter $Z$ and $(k, Z)$ curves of panels consisting of different materials will be constructed (both $k$ and $Z$ are defined in section 3 of this article).

\section{Finite element modeling procedure}

In this study, we carry out a three-dimensional FEM procedure in order to calculate the critical buckling load and the corresponding mode shape of the considered panels subjected to uniform axial compressive loading. The panels consist of GLARE, UD composite laminates or monolithic aluminum. Since the panels are thin, SHELL 181 elements are used for their modeling [5]. The behavior of the constituent materials of GLARE panels, aluminum and UD composite, is idealized using isotropic and orthotropic linear elastic material models. A line pressure load $N_{z}$, which is always equal to unity, is applied along the two curved edges, in order to simulate the uniaxial compressive stress $\sigma_{z}$ as shown in Fig. 1.

Using the eigenvalue buckling analysis in accordance with ANSYS recommendations, the critical buckling load $N_{z c}$ and the corresponding mode shape are predicted. With respect to the FE discretization, the convergence of the numerical results is always verified by comparison of results corresponding to fine and very fine panel mesh density. It is noted that the von Mises stresses in aluminum layers and the stress components in composite layers are always well below the proportional limit of aluminum and the composite ply strengths, respectively, indicating that elastic bucking occurs.

The curvature parameter $Z$ of aluminum panels is calculated by [6]:

$$
Z=\frac{b^{2}}{R t} \sqrt{1-v^{2}}
$$

where $v$ is the Poisson's ratio for 2024-T3 aluminum.

The curvature parameter of laminated panels is calculated by $[1,7]$ :

$$
Z=\frac{b^{2}}{R} \sqrt{\frac{A_{11} A_{22}-A_{12}^{2}}{12 \sqrt{A_{11} A_{22} D_{11} D_{22}}}}
$$

where $A_{i j}$ and $D_{i j}(i, j=1,2)$ are the extensional and bending stiffnesses of the laminate.

The buckling coefficient of the aluminum panels is calculated by [3]:

$$
k=\frac{N_{z c} b^{2}}{\pi^{2} D}
$$

where $D$ is the bending stiffness of the isotropic panel.

The buckling coefficient of laminated panels is calculated by [8]:

$$
k=\frac{N_{z c} b^{2}}{\pi^{2} \sqrt{D_{11} D_{22}}}
$$

The $(k, Z)$ curve of a considered material is obtained using FEM models with variable curvature parameter $Z$. The length and the thickness of the panels are constant: $L=1 \mathrm{~m}$ and $t$ $=1.875 \mathrm{~mm}$. The width $b$ and the radius of curvature $R$ of the panels vary: $0.5 \mathrm{~m} \leq b \leq 0.8$ $m$ and $0.31 m \leq R \leq 12 m$ so that the curvature parameter is approximately greater than 10 and lower than 1100. The range of $Z$ between 10 and 1000 for isotropic panels is 
characterized by a transition from flat plate to complete cylinder behavior [1,9]. It is noted that the variable panel dimensions are identical for all analyzed materials.

The applied classically simply supported bc are analyzed by Timoshenko \& Gere [3]: the radial displacements become zero along the edges of the panel, the axial displacements become zero along the straight edges and the circumferential displacements become zero along the curved edges. The coordinate system is orientated as shown in Fig.1. The presented FEM modeling procedure is applied to the following GLARE grades:

GLARE $2 \mathrm{~A}-3 / 2-0.458,\left[\mathrm{Al} / 0^{\circ} \mathrm{gl} / 0^{\circ} \mathrm{gl} / \mathrm{Al} / 0^{\circ} \mathrm{gl} / 0^{\circ} \mathrm{gl} / \mathrm{Al}\right]$

GLARE 3-3/2-0.458, $\left[\mathrm{Al} / 0^{\circ} \mathrm{gl} / 90^{\circ} \mathrm{gl} / \mathrm{Al} / 90^{\circ} \mathrm{gl} / 0^{\circ} \mathrm{gl} / \mathrm{Al}\right]$

GLARE 4B-3/2-0.375, $\left[\mathrm{Al} / 90^{\circ} \mathrm{gl} / 0^{\circ} \mathrm{gl} / 90^{\circ} \mathrm{gl} / \mathrm{Al} / 90^{\circ} \mathrm{gl} / 0^{\circ} \mathrm{gl} / 90^{\circ} \mathrm{gl} / \mathrm{Al}\right]$

GLARE 5-3/2-0.292, $\left[\mathrm{Al} / 0^{\circ} \mathrm{gl} / 90^{\circ} \mathrm{gl} / 90^{\circ} \mathrm{gl} / 0^{\circ} \mathrm{gl} / \mathrm{Al} / 0^{\circ} \mathrm{gl} / 90^{\circ} \mathrm{gl} / 90^{\circ} \mathrm{gl} / 0^{\circ} \mathrm{gl} / \mathrm{Al}\right]$

GLARE $2 / 1-0.438,\left[\mathrm{Al} / 0^{\circ} \mathrm{gl} / 90^{\circ} \mathrm{gl} / 90^{\circ} \mathrm{gl} / 0^{\circ} \mathrm{gl} / 0^{\circ} \mathrm{gl} / 90^{\circ} \mathrm{gl} / 90^{\circ} \mathrm{gl} / 0^{\circ} \mathrm{gl} / \mathrm{Al}\right]$

It is also applied to the following symmetric UD glass-epoxy composite laminates: $\left[0^{\circ}\right]_{15},\left[0^{\circ} / 90^{\circ} / 0^{\circ} / 90^{\circ} / 0^{\circ} / 90^{\circ} / 0^{\circ} / \overline{90^{\circ}}\right]_{\mathrm{s}}$ and to $2024-\mathrm{T} 3$ aluminum panels. The $0^{\circ}$ fiber orientation angle is parallel with the length $L$ and the z-axis of the global cylindrical coordinate system, which is always located as shown in Fig. 1. The last number of the aforementioned coded name of GLARE panels indicates the thickness of aluminum layers in $\mathrm{mm}$. Each ply of the examined GLARE and composite laminates consisting of S2-glass UD fiber prepreg has a thickness of $0.125 \mathrm{~mm}$. The material properties of aluminum and S2-glass UD prepreg layers considered for our calculations can be found in reference [10].

\section{Comparison of FEM with analytical results}

We have initially compared the FEM results with corresponding theoretical results obtained using a previously developed analytical model, for monolithic aluminum panels, in order to validate our FEM procedure and the suitability of the applied classically simply supported bc.

When the squares of $t^{2} /\left(12 R^{2}\right)$ and $N\left(1-v^{2}\right) /(E t)$ are small quantities, the buckling load of complete cylinders under uniform axial compression is given by $[3,11]$ :

$$
\begin{gathered}
N=\frac{E t}{1-v^{2}} \frac{\varphi}{\psi} \\
\varphi=\left(1-v^{2}\right) \beta^{4}+\frac{t^{2}}{12 R^{2}}\left[\left(n^{2}+\beta^{2}\right)^{4}-(3-v)(2+v) n^{2} \beta^{4}+\right. \\
\left.2\left(1-v^{2}\right) \beta^{4}-(7+v) n^{4} \beta^{2}+(3+v) n^{2} \beta^{2}+n^{4}-2 n^{6}\right] \\
\psi=\beta^{2}\left\{\left(n^{2}+\beta^{2}\right)^{2}+\left(n^{2}+\frac{2}{1-v} \beta^{2}\right)\left[1+\frac{t^{2}}{12 R^{2}}\left(n^{2}+\beta^{2}\right)^{2}\right]-\frac{2 v^{2} \beta^{2}}{1-v}+\right. \\
\left.\frac{t^{2}}{12 R^{2}}\left(n^{2}+\frac{2}{1-v} \beta^{2}\right)\left[n^{2}+(1-v) \beta^{2}\right]\right\}
\end{gathered}
$$

where $E$ is the Young's modulus, $m$ and $n$ are the numbers of half-waves in the longitudinal and circumferential direction of the shell respectively and $\beta=(m \pi R) / L$.

Equations (5)-(7) are valid for isotropic cylindrical panels as well; the only change is $n$ being replaced by $n \pi / \varphi$. Consequently, for variable numbers of half-waves $m$ and $n$, the minimum value of $N$ can be obtained which is equal to the critical buckling load of the considered panel. Two validation models have been used and their geometry is determined as follows: validation model (a) with $R=1.30 \mathrm{~m}, b=0.22 \mathrm{~m}, L=0.33 \mathrm{~m}$ and validation model (b) with $R=0.65 \mathrm{~m}, b=0.22 \mathrm{~m}, L=0.30 \mathrm{~m}$. The thickness of these panels is equal to $1.875 \mathrm{~mm}$. A very good agreement between our FEM results and the analytical predictions of $N_{z c}$ can be observed from Table 1 for the aluminum panels, since their 
deviation is small. Additionally, for the considered panels, the numbers of longitudinal $(m)$ and circumferential $(n)$ half-waves of the FEM buckled mode shape agree with analytical predictions. These facts demonstrate the validity of the implemented FEM modeling procedure and the applied bc. The same validated bc are also used for the composite panels and FMLs.

Table 1. FEM versus analytical values of critical buckling load of simply supported aluminum, UD $\left[0^{\circ}\right]_{15}$ composite and GLARE $2 \mathrm{~A}-3 / 2-0.458$ panels.

\begin{tabular}{|c|c|c|c|c|c|c|}
\hline Material & $\begin{array}{c}\text { Validation } \\
\text { Model }\end{array}$ & $\mathrm{m}$ & $\mathrm{n}$ & $\begin{array}{c}\mathrm{N}_{\mathrm{zc}} \\
\text { Analytical } \\
{[\mathrm{N} / \mathrm{m}]}\end{array}$ & $\begin{array}{c}\mathrm{N}_{\mathrm{zc}} \\
\mathrm{FEM} \\
{[\mathrm{N} / \mathrm{m}]}\end{array}$ & $\begin{array}{c}\mathrm{N}_{\mathrm{zc}} \\
\text { deviation } \\
(\%)\end{array}$ \\
\hline \multirow{2}{*}{$\begin{array}{c}\text { Aluminum 2024- } \\
\text { T3 }\end{array}$} & $\mathrm{a}$ & 3 & 1 & 120747.9 & 126748.1 & +4.97 \\
\hline & $\mathrm{b}$ & 5 & 1 & 243913.4 & 230471.0 & -5.51 \\
\hline \multirow{2}{*}{$\begin{array}{l}\mathrm{UD}\left[0^{\circ}\right]_{15} \text { glass- } \\
\text { epoxy composite }\end{array}$} & $\mathrm{a}$ & 2 & 1 & 29653.9 & 29341.1 & -1.06 \\
\hline & $\mathrm{b}$ & 2 & 2 & 55209.5 & 58806.1 & +6.51 \\
\hline \multirow{2}{*}{$\begin{array}{c}\text { GLARE } 2 \mathrm{~A}-3 / 2- \\
0.458\end{array}$} & $\mathrm{a}$ & 3 & 1 & 103348.2 & 107646.5 & +4.16 \\
\hline & $\mathrm{b}$ & 4 & 1 & 208716.3 & 196720.1 & -5.75 \\
\hline
\end{tabular}

Pertinent literature results have been used for comparison with our FEM results in order to validate our FEM modeling of FML and composite panels. In reference [1] a simple oneterm trigonometric representation for the buckled shape is used and as a result a closedform solution is obtained, which satisfies classically simply supported bc and is applicable to thin specially orthotropic laminated panels:

$$
\sigma=\frac{1}{\alpha^{2} t}\left(\frac{\alpha^{4}}{R^{2} A_{1}}+D_{1}\right)
$$

where $\alpha=m \pi / L$ and:

$$
\begin{aligned}
& A_{1}=A_{11}^{*} \gamma^{4}+A_{22}^{*} \alpha^{4}+\left(2 A_{12}^{*}+A_{66}^{*}\right) \alpha^{2} \gamma^{2} \\
& D_{1}=D_{11} \alpha^{4}+D_{22} \gamma^{4}+2\left(D_{12}+2 D_{66}\right) \alpha^{2} \gamma^{2}
\end{aligned}
$$

where $A_{i j}^{*}$ is the $\mathrm{ij}^{\text {th }}$ element of the inverted extensional stiffness matrix, $\gamma=n \pi / b$ and $\sigma=N_{z} / t$.

Using equations (4) and (8) the critical buckling load and the buckling coefficient $k$ can be calculated. Again, for variable numbers of half-waves $m$ and $n$, the minimum value of $\sigma$ can be obtained which is equal to the critical buckling stress of the considered panel.

The dimensions of the panels which have been used for validation of the implemented FEM procedure in the cases of FMLs and composites are identical with those of aluminum panels as shown in Table 1. A very good agreement between our FEM results and the analytical predictions of $N_{z c}$ can be observed for UD $\left[0^{\circ}\right]_{15}$ glass-epoxy composite and GLARE 2A-3/2-0.458 panels from Table 1. Furthermore, the numbers of half-waves of each FEM calculated critical mode shape agree with analytical predictions. In Fig. 2 the deflections of a representative mode shape corresponding to the critical buckling load of validation model (b) for UD glass-epoxy with $0^{\circ}$ fiber orientation angle are illustrated. It is seen from Fig. 2 that the number of longitudinal half-waves is equal to the number of circumferential half-waves $(m=n=2)$, as also predicted from the analytical calculations. We have verified using the aforementioned geometries of the two validation models, that the corresponding numerical and analytical values of $N_{z c}$ are in very good agreement for the cross-ply UD composite and all remaining GLARE grades and that numerical and analytical values of $m, n$ always coincide. Based on these comparisons the implemented FEM procedure for FMLs and composite panels is validated. 


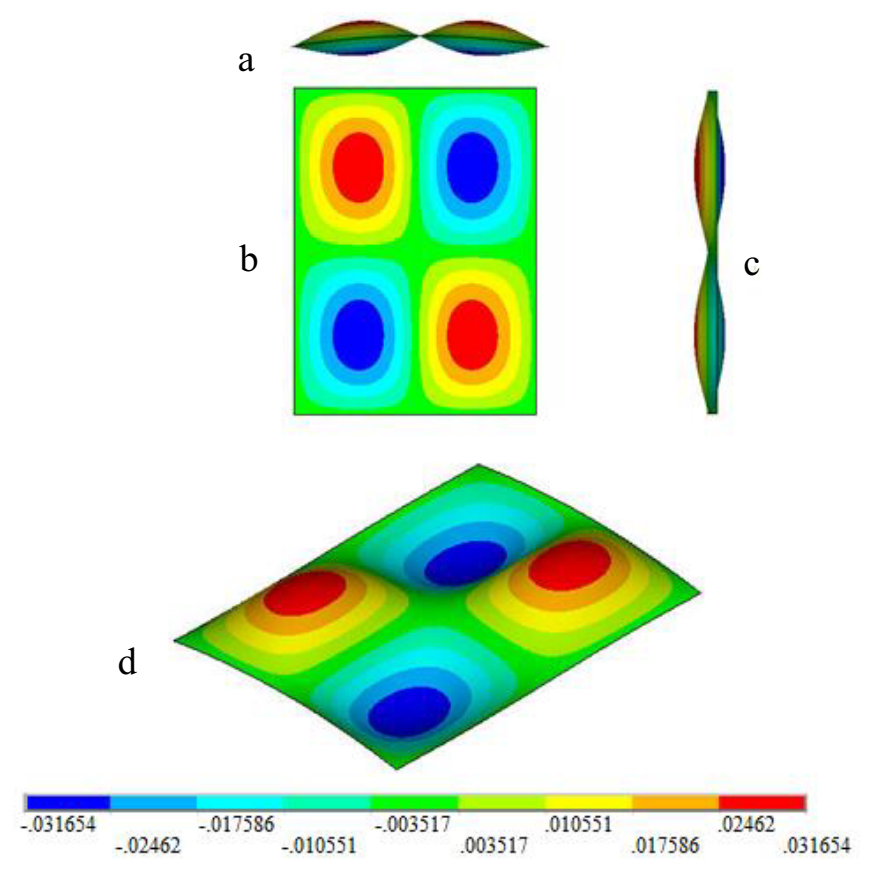

Fig. 2. FEM top view (b) along with back (a), side (c) views and isometric view (d) contours of the deflections corresponding to the critical chessboard buckling mode shape of a simply supported UD glass-epoxy panel with $0^{\circ}$ fiber orientation angle under uniform axial compression $(L=0.3 \mathrm{~m}, b=$ $0.22 \mathrm{~m}, R=0.65 \mathrm{~m})$.

\section{Results and discussion}

In Fig. 3 the calculated $(k, Z)$ diagrams corresponding to the analyzed GLARE 3-3/2-0.458, composite and aluminum panels are given for classically simply supported bc. The buckling load of the panels can be directly obtained using these diagrams in combination with equations (3) and (4), for a specific curvature parameter. It can be observed from Fig. 3, which is presented in double logarithmic scale, that the trend of the $(k, Z)$ curves is similar in all cases. Specifically, the buckling coefficient increases for increasing curvature parameter, regardless of the material. We have verified that the $(k, Z)$ curves are approximately linear in double logarithmic scale for all considered materials. Consequently, $k$ can be expressed as a function of $Z$ as follows:

$$
\log k \approx \mu \log Z+\log \lambda
$$

where $\lambda$ and $\mu$ are constants.

In double linear scale equation (11) is equivalent with the following expression:

$$
k \approx \lambda Z^{\mu}
$$

We have implemented a nonlinear regression using equation (12) of the calculated $(k, Z)$ data points in double linear scale (which yields a linear $(k, Z)$ curve in double logarithmic scale) for each analyzed material and Table 2 summarizes the $\lambda, \mu$ values along with the coefficients of determination $\mathrm{R}^{2}$. It can be observed from Table 2 that the $\mathrm{R}^{2}$ values are close to unity in all examined cases. This fact indicates that the function $k=\lambda Z^{\mu}$ is a 
reliable trendline. Using the $\lambda, \mu$ values of Table 2 in combination with equation (12), the buckling coefficient of cylindrical panels consisting of the considered materials can be easily approximated satisfactorily for classically simply supported bc and $10 \leq Z \leq 1100$.

Table 2. Parameters and $\mathrm{R}^{2}$ values of the $(k, Z)$ trendlines for simply supported cylindrical panels under uniaxial compression consisting of different materials.

\begin{tabular}{|c|c|c|c|}
\hline Material & $\boldsymbol{\lambda}$ & $\boldsymbol{\mu}$ & $\mathbf{R}^{\mathbf{2}}$ \\
\hline 2024-T3 & 0.8650 & 0.9487 & 0.9963 \\
\hline UD glass-epoxy 0 $^{\circ}$ & 0.6777 & 0.9628 & 0.9999 \\
\hline UD glass-epoxy cross-ply $^{-}$ & 0.6508 & 0.9389 & 0.9995 \\
\hline GLARE 2A-3/2-0.458 & 0.8425 & 0.9475 & 0.9965 \\
\hline GLARE 3-3/2-0.458 & 0.8731 & 0.9461 & 0.9968 \\
\hline GLARE 4B-3/2-0.375 & 0.8584 & 0.9472 & 0.9965 \\
\hline GLARE 5-3/2-0.292 & 0.8372 & 0.9475 & 0.9971 \\
\hline GLARE 2/1-0.438 & 0.8618 & 0.9424 & 0.9962 \\
\hline
\end{tabular}

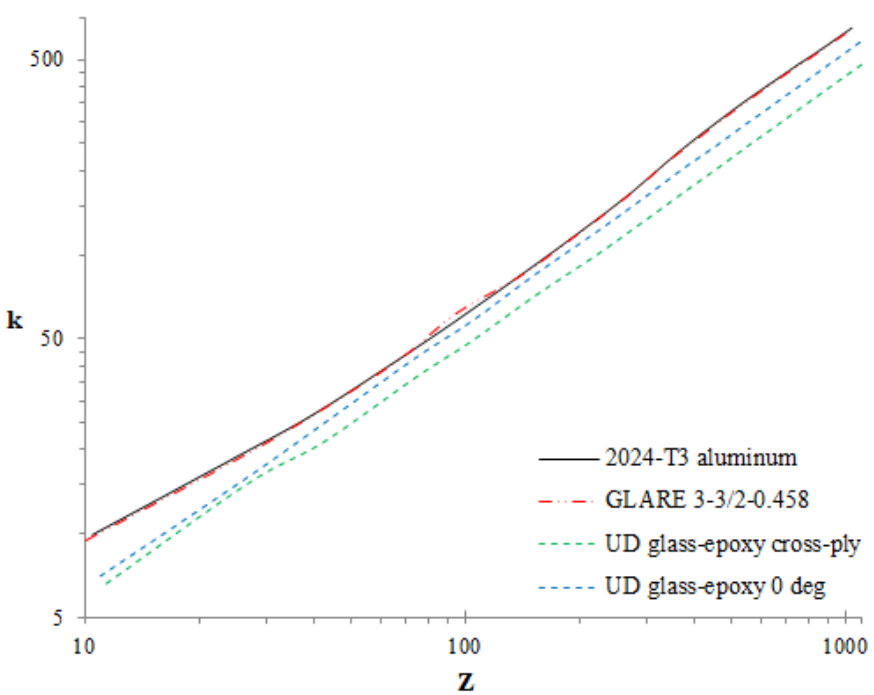

Fig. 3. Buckling coefficients of GLARE 3-3/2-0.458, aluminum and UD composite cylindrical panels with simply supported bc under axial compression.

It can also be observed from Fig. 3 that the $(k, Z)$ curve of GLARE 3 follows the trend of aluminum $(k, Z)$ curve very closer than the trend of the cross-ply composite $(k, Z)$ curve. This observation which is valid for all GLARE grades considered in this article indicates the domination of aluminum in the buckling response of GLARE panels, regardless the fact that the Metal Volume Fraction (MVF) is less than 0.5 for the GLARE 5 and the GLARE $2 / 1$ panels. Additionally, from Fig. 3 it is observed that the differentiation between crossply and $0^{\circ}$ fiber orientation has a considerable effect on the behavior of the $(k, Z)$ curves in the case of UD composite laminates, whereas in the case of GLARE 3 and GLARE 2A panels this effect of the fiber orientation angle is found to be small.

\section{Conclusions}

This article deals with the elastic buckling of simply supported cylindrical GLARE panels subjected to uniaxial compression. Classically simply supported boundary conditions are considered. The finite element method is employed in combination with the eigenvalue 
buckling analysis in order to predict the buckling coefficient of the laminates and obtain relevant useful diagrams for engineering calculations. Five different GLARE grades are analyzed along with two UD glass-epoxy composites and monolithic 2024-T3 aluminum.

It is found that aluminum has a stronger impact on the buckling behavior of the GLARE panels than the composite layers. From the buckling coefficient-curvature parameter diagrams of simply supported panels in double logarithmic scale, it is found that there is an approximately linear relation between these two parameters. Based on this finding, appropriate regressions are implemented in order to derive approximate analytical formulas of the buckling coefficient as a function of the curvature parameter for the considered materials.

\section{References}

1. K. Verolme, The initial buckling behaviour of flat and curved fiber metal laminate panels, Report LR-785, Delft University of Technology, Netherlands (1995)

2. T. Sinmazçelik, E. Avcu, M.O. Bora, O. Çoban, Mater. Des. 32, 3671-3685 (2011)

3. S.P. Timoshenko, J.M. Gere, Theory of elastic stability, Mc-Graw Hill, $2^{\text {nd }}$ ed. (1961)

4. J.L. Verolme, The development of a design tool for fiber metal laminate compression panels, Ph.D. Dissertation, Delft University of Technology, Netherlands (1995)

5. ANSYS, Structural Analysis Guide, ANSYS Inc. (2014)

6. S.B. Batdorf, A simplified method of elastic-stability analysis for thin cylindrical shells, Report no. 874, NACA, USA (1947)

7. M.P. Nemeth, Nondimensional parameters and equations for buckling of symmetrically laminated thin elastic shallow shells, TM-104060, NASA, USA (1991)

8. D. Gay, S.V. Hoa, S.W. Tsai, Composite materials design and applications, CRC Press LLC (2003)

9. G. Gerard, H. Becker, Handbook of structural stability part III-buckling of curved plates and shells, TN-3783, NACA, USA (1957)

10. A.S. Yaghoubi, B. Liaw, Int. J. Impact Eng. 54, 138-148 (2013)

11. C.M. Wang, C.Y. Wang, J.N. Reddy, Exact solutions for buckling of structural members, CRC Press LLC (2005) 